\title{
INCIDENCIA DEL TRABAJO INFANTIL EN EL DESARROLLO DE LA VIOLENCIA DE GÉNERO EN ECUADOR
}

\section{Raúl Carpio Freire}

rcarpiof@uees.edu.ec

Universidad Espíritu Santo (Ecuador)
Katherine Valdiviezo Cantos

kvaldiviezo@uees.edu.ec

Universidad Espíritu Santo (Ecuador)

Recibido: 06-09-2014

Aceptado: 21-04-2015

\section{Resumen}

Actualmente la problemática del trabajo infantil y la violencia de género constituyen un tema de repercusión política y social para el Ecuador. El presente artículo busca determinar si el trabajo infantil es una variable que influye o resulta en maltrato de género; para lo cual se realizó un estudio de regresión lineal al total de niños y niñas y de mujeres ecuatorianas que habitan en las diferentes provincias del Ecuador y que presentan ambas características. De esta manera se busca determinar la relación que existe entre ambas; con el fin de poder plantear propuestas que conlleven a soluciones alternativas para la disminución de este flagelo.

Palabras clave: Maltrato de género, violencia a la mujer, trabajo infantil, niños/as ecuatorianos, mujeres ecuatorianos, estudio de regresión lineal, problemas sociales.

\begin{abstract}
Currently, the problem of child work and gender violence constitutes a matter of political and social impact for Ecuador. This article seeks to determine whether child labor is or could be considered as a variable that influences or generate gender abuse; for which, at first, a linear regression study was performed to all Ecuadorian children and women living in the different provinces of the country and that have both characteristics. Thus, this analysis observes the relation between both variables, in order to make proposals that lead to alternative solutions for reducing this scourge.
\end{abstract}

Keywords: gender abuse, violence to women, child labor, Ecuadorian children, Ecuadorian women, linear regression study, social problems. 


\section{Introducción}

El trabajo infantil y la violencia contra la mujer son dos problemáticas sociales que afrontan muchos países en el mundo, en especial los de Latinoamérica, porque son los países donde aún se encuentran muchos grupos de personas y sociedades dominadas por ideas masculinas, las cuales tienden a tener repercusiones sobre la agresión de género; esos mismos grupos se presentan indiferentes a usufructuar de los menores de edad para su bien propio.

El presente artículo plantea una la siguiente interrogante: “¿Representa el trabajo infantil una variable que influye en el futuro a que suscite la violencia contra la mujer?", por ende se enfocará en el estudio de estas dos variables que gobiernan los problemas en los que está inmerso el Ecuador, el trabajo infantil y la violencia de género; a las cuales se les realizará el análisis pertinente para proporcionar una respuesta a la interrogante planteada. Los datos a utilizar serán obtenidos de las bases de datos del Instituto Ecuatoriano de Estadísticas y Censos (INEC) de las encuestas realizadas a estos grupos de mujeres y niños ecuatorianos en el año 2012.

\section{Marco Teórico}

El trabajo infantil es considerado como el acto en el que un adulto, ya sea un familiar o un tercero, obliga a un menor de edad entre 5 a 14 años a ejercer actividades varias contra su voluntad para obtener ingresos, de los cuales el niño no percibe en su mayoría, además de privarlo de su infancia (Humanium, 2014). El trabajo infantil es catalogado según el Fondo de las Naciones Unidas para la Infancia "como cualquier trabajo que supere una cantidad mínima de horas, dependiendo de la edad del niño o niña y de la naturaleza del trabajo" (UNICEF, s.f.). Este tipo de trabajo se considera perjudicial para la infancia del menor y por tanto debería erradicarse, no es su mayoría, pero en su totalidad. Gran parte de estas actividades suelen ser ilícitas y van contra la ley del país y contra el Código de la niñez y de la adolescencia, puesto que viola los derechos de los menores de edad; además de privarlos de su libertad para llevar una vida normal y les niega el acceso a una escolarización digna; atenta contra su desarrollo 
psicológico y social, contra su dignidad y le provoca estrés social, psicológico y físico por la gran responsabilidad que se le impone a los menores a tan corta edad.

Muchas de las actividades que los obligan a ejercer van desde vender objetos o caramelos en las calles, recolección de objetos en los basurales, robo, limpieza de automóviles en los semáforos, eh incluso pueden llegar a ser perjudiciales y llevar a los niños a la prostitución y al alquiler de los mismos a terceros por una pequeña comisión diaria, a cambio de utilizarlos como objetos para pedir limosnas en las calles. Todas estas actividades dejan expuestos a los menores a una infinidad de peligros en el diario vivir, tales como: asaltos, robos, violaciones sexuales, pandillas, drogas, trata de blancos, entre otros. El trabajo infantil afecta no solo al desarrollo económico del país, sino también al desarrollo social del mismo por los crecientes niveles de pobreza y deserción escolar que trae consigo, igualmente de las altas tasas de analfabetismo. Para Alison Vásconez el trabajo infantil se lo define como:

"El trabajo infantil representa, en el nivel macro, un retraso económico y social; económico porque presiona los niveles salariales y el empleo adulto a la baja; social, porque priva a la sociedad del futuro del capital humano que requiere desarrollo sostenido. En lo micro, provoca la reproducción intergeneracional de la pobreza, desigualdad y carencias de activos básicos para el desarrollo personal" (Vasconez, 2003).

En el Ecuador se ha estimado un mínimo de edad para que los niños pueda trabajar, y esta es la edad es a partir de los 15 años; sin embargo en el estudio realizado por la INEC sobre el trabajo infantil en el país se engloba a niños con edades que va desde los 5 hasta los 17 años; puesto que la mayoría de edad se la reconoce a las 18 años. En el 2011 el gobierno llevo a cabo un programa para erradicar el trabajo infantil en los basurales y logro retirar cerca de 2,160 niños de este trabajo peligroso, posicionando al país entre el primero de Latinoamérica por haber abolido esta forma de trabajo inhumana.

Son muchos los factores que engloban el trabajo infantil y que están fuertemente ligados para que esta actividad siga aun en pie, entre los más relevantes se pueden destacar: la pobreza, la discriminación y la globalización; siendo este último uno de los más recientes, puesto que las empresas han comenzado a buscar diversas formas y métodos para poder reducir sus costes de mano de obra y de esa manera poder introducirse a competir en el mercado internacional con el resto de industrias, lo que los conlleva a la contratación de menores de edad, como es el 
caso de las bananeras en el Ecuador. El trabajo infantil es un mal presente en el país que se lo puede palpar en las calles, en las paradas de buses, en el tráfico y muchos otros sitios a día a día.

Tabla 1. Porcentajes y número de niños que se encuentran laborando en las diferentes regiones del Ecuador

\begin{tabular}{|c|c|c|c|c|}
\hline & & $\begin{array}{c}\text { Trabajo } \\
\text { infantil total }\end{array}$ & $\begin{array}{c}\text { Población total } \\
\text { niños }\end{array}$ & $\begin{array}{c}\text { Tasa de trabajo } \\
\text { infantil }\end{array}$ \\
\hline \multirow[t]{19}{*}{ Dominios } & Cotopaxi & 32.053 & 127.503 & $25,14 \%$ \\
\hline & Bolívar & 12.996 & 58.424 & $22,24 \%$ \\
\hline & Chimborazo & 28.769 & 136.894 & $21,02 \%$ \\
\hline & Cañar & 12.354 & 71.084 & $17,38 \%$ \\
\hline & Loja & 21.417 & 134.142 & $15,97 \%$ \\
\hline & Azuay & 31.930 & 206.483 & $15,46 \%$ \\
\hline & Amazonía & 33.671 & 259.381 & $12,98 \%$ \\
\hline & Tungurahua & 14.416 & 134.985 & $10,68 \%$ \\
\hline & Carchi & 4.838 & 46.713 & $10,36 \%$ \\
\hline & Imbabura & 10.799 & 117.895 & $9,16 \%$ \\
\hline & Total & 359.597 & 4.199 .296 & $8,56 \%$ \\
\hline & Esmeraldas & 12.898 & 179.711 & $7,18 \%$ \\
\hline & Santo Domingo & 8.064 & 15.987 & $6,95 \%$ \\
\hline & Los Ríos & 13.286 & 238.979 & $5,56 \%$ \\
\hline & Guayas & 54.027 & 1.011 .284 & $5,34 \%$ \\
\hline & Pichincha & 35.410 & 674.481 & $5,25 \%$ \\
\hline & El Oro & 8.552 & 170.898 & $5,00 \%$ \\
\hline & Santa Elena & 4.613 & 94.537 & $4,88 \%$ \\
\hline & Manabí & 17.818 & 408.711 & $4,36 \%$ \\
\hline
\end{tabular}

Extraída del (Instituto Ecuatoriano de Estadísticas y Censos, 2012)-Encuesta Nacional de Trabajo Infantil

Por otro lado, la violencia contra la mujer se la define como el acto en el que una persona del género masculino, ya sea un familiar, un conviviente o un tercero, atenta contra la integridad de una persona del género femenino, ocasionándole de esta forma diversos 
trastornos psicológicos y atentando contra su integridad física. La Organización de las Naciones Unidas (ONU) define la violencia contra la mujer como:

"Todo acto de violencia basado en la pertenencia al sexo femenino que tenga o pueda tener como resultado un daño o sufrimiento físico, sexual o psicológico para la mujer, así como las amenazas de tales actos, la coacción o la privación arbitraria de la libertad, tanto si se producen en la vida pública como en la vida privada" (Organización de las Naciones Unidas, 1993).

En el Ecuador, la violencia contra la mujer es uno de los tantos males sociales con los que un gran número de mujeres tienen que convivir diariamente. Este tipo de violencia abarca más factores, como el abuso sexual, psicológico y patrimonial; haciendo referencia a este último término "Se refiere a cualquier acto que afecte o atente contra el patrimonio o supervivencia de la víctima, como la sustracción o retención de objetos, documentos o recursos económicos” (Instituto Ecuatoriano de Estadísticas y Censos, 2012).

Haciendo referencia a los demás términos; violencia física engloba todo acto que cause daño, sufrimiento o dolor físico a la persona que ha sido objeto de la agresión; la violencia psicológica hace referencia a toda acción que cause dolor o perturbación emocional, alteración psicológica o disminución de la autoestima en el individuo agredido, esta forma de maltrato suele ser presentada en gritos, insultos, prohibiciones, humillaciones, entre otros "Esta forma de violencia es muy perjudicial porque además de que es progresiva, en ocasiones logra causar daños irreversibles en la personalidad del agredido" (Consejo Nacional de Población , 2012). La violencia sexual, por otro lado, hace referencia al abuso sexual o la imposición de acciones sexuales a un individuo, obligándole a mantener relaciones o prácticas sexuales contra su voluntad mediante el uso de fuerza física, intimidación o amenazas.

La violencia de género se funda a través de ese "sistema social de patriarcado, el cual integra un mensaje que asevera que los poderosos se hallan en su derecho de dominar a los menos poderosos y que la violencia se contempla como una herramienta válida y necesaria para ello" (Expósito, 2005). Por otro lado, la mujer que sufre de maltratos, es capaz de desarrollar el síndrome del estrés postraumático, en el caso de haber sufrido violencia sexual las probabilidades de padecerlo pueden crecen en un $50 \%$ a $60 \%$; este síndrome viene 
acompañado de síntomas como depresión, ansiedad, inadaptación en diferentes áreas de la vida y baja autoestima.

La violencia de género es una degradación a los derechos de las mujeres y una violación a sus derechos humanos y es considerada como un gran problema para alcanzar la igualdad y la equidad de género en el Ecuador. En los años 90 solo se hacía referencia a la violencia intrafamiliar; sin embargo en 1995 en el Ecuador se promulga la primera ley contra la violencia de la mujer y de la familia; y en el año 2007 el gobierno declara una política para erradicar la violencia de género.

Tabla 2. Porcentajes y número de mujeres que han sufrido violencia de género en las diferentes regiones del Ecuador

\begin{tabular}{|c|c|c|c|}
\hline PROVINCIA & POBLACIÓN MUJERES & PORCENTAJE & TOTAL \\
\hline Santa Elena & 151.831 & $47,10 \%$ & 71.512 \\
\hline Manabí & 680.481 & $48,30 \%$ & 328.672 \\
\hline Amazonía & 357.501 & $63,90 \%$ & 228.443 \\
\hline Carchi & 83.369 & $59,10 \%$ & 49.271 \\
\hline Guayas & 1.829 .569 & $56,60 \%$ & 1.035 .536 \\
\hline Los Ríos & 380.016 & $57,30 \%$ & 217.749 \\
\hline Chimborazo & 239.180 & $57,40 \%$ & 137.289 \\
\hline Esmeraldas & 262.780 & $58,70 \%$ & 154.252 \\
\hline El Oro & 296.297 & $58,80 \%$ & 174.223 \\
\hline Loja & 228.172 & $58,80 \%$ & 134.165 \\
\hline Bolívar & 93.766 & $60,30 \%$ & 56.541 \\
\hline Cotopaxi & 210.580 & $60,70 \%$ & 127.822 \\
\hline Imbabura & 204.580 & $63,50 \%$ & 129.908 \\
\hline Cañar & 119.949 & $76,40 \%$ & 91.641 \\
\hline Azuay & 375.083 & $68,80 \%$ & 258.057 \\
\hline Pichincha & 1.320 .576 & $71,20 \%$ & 940.250 \\
\hline Tungurahua & 259.800 & $70,80 \%$ & 183.938 \\
\hline Santo Domingo & 184.955 & $59,00 \%$ & 109.123 \\
\hline
\end{tabular}

Tabla elaborada por la autora; datos extraídos de (Instituto Ecuatoriano de Estadisticas y Censos, 2012)- Encuesta nacional de relaciones familiares y violencia de género contra las mujeres. 
Según los resultado de las encuestas realizadas por el INEC en el año 2012, alrededor del 8,6\% de los niños ecuatorianos entre 5 a 17 años trabajan a nivel territorial, las provincias donde se puede encontrar mayor porcentaje de trabajo infantil son las provincias del Cotopaxi, Chimborazo y Bolívar con 25,1\%, 21\% y 22,2\% respectivamente; a nivel de etnicidad los niños indígenas son los que más trabajan donde se puede destacar un $29 \%$, dato muy relevante en la investigación puesto que a nivel de etnicidad también se encuentran las mujeres indígenas con la tasa porcentual más alta en cuanto a violencia de género con un 67,8\%. En el Ecuador 6 de cada 10 mujeres han sufrido violencia de género, lo que equivale a más del $50 \%$ de la población femenina del país.

Actualmente no existen estudios que se hayan enfocado en analizar la posible relación en estas dos variables, por ende el presente paper pretende demostrar si el trabajo infantil tiene repercusiones futuras en los niños que han sufrido de estos abusos y se vea reflejado en la violencia de género contra sus esposas o en el caso de las niñas, se vea reflejado en su baja autoestima y las haga sentir como objeto de abusos continuo.

\section{Metodología}

El área de estudio es el Ecuador, el cual cuenta con una población aproximada de 16.042.020 millones de personas, dentro de las cuales la población total niños/as y adolescentes entre los 5 a 17 años de edad asciende a 4.199.296 niños/as, de estas cifras el 2.139.737 son de sexo masculino y 2.059.559 son de sexo femenino; de ellos el 8.56\% son obligados a realizar trabajados forzados dejando un total de 359.597 niños/as ecuatorianos, de igual manera estas cifras se dividen en $10.55 \%$ de niños y $6.49 \%$ de niñas, dejando un total de 225.846 niños y 133.751 niñas que son explotados y en la mayoría de los casos se los obliga a laborar contra su voluntad.

De igual manera la población total de mujeres en el país es de7.305.816 millones, de las cuales el $39.40 \%$ de ellas ha sufrido algún tipo de violencia, dejando un total de 2.878 .492 millones de mujeres que han sufrido agresiones o han sido maltratadas por sus cónyuges o terceros. El estudio comprende todas las provincias del Ecuador, dejando al margen las zonas no delimitadas y la provincia de Galápagos, de igual manera bajo la nomenclatura "Amazonia" 
se resumieron las provincias de Orellana, Pastaza, Sucumbíos, Napo, Zamora y Morona debido a que en estas provincias no se encuentran poblaciones tan grandes como en las demás.

El siguiente estudio es de carácter cuantitativo, puesto que se contará con datos de carácter numérico extraídos de las bases de datos de INEC y se analizara la relación entre las dos variables respectivamente. La población escogida serán los niños/as y las mujeres ecuatorianas de las principales provincias del país y la muestra serán todos los niños y niñas ecuatorianos que hayan sufrido de trabajo infantil y todas las mujeres que hayan sufrido algún tipo de violencia de género.

El método que se utilizará para este estudio es el método de regresión lineal para encontrar la relación que tiene la variable dependiente $\mathrm{Y}$ (violencia de género) o también conocida como "respuesta" con la variable independiente X (trabajo infantil) también conocida como variable "predicción" y de esa manera poder medir el grado de asociación entre las dos variables y poder realizar predicciones de la variable dependiente.

En este estudio se realizaran tres análisis diferentes, el primero se enfocara en identificar si el maltrato infantil si tiene relación o si influye en cierto grado con el maltrato de género, para lo cual se analizara con el método de regresión lineal el número de niños/as que son obligados a trabajar con el número de mujeres maltratas por provincia; mientras que el segundo y el tercer análisis se enfocara en determinar que género influye o tiene más relación con el maltrato de género, para lo cual se analizara por separado el total de niños varones que han sido víctimas del trabajo infantil con el número total de mujeres ecuatorianas que han sufrido de violencia de género, de igual manera se realizara un tercer análisis con el total de niñas/adolescentes que han padecido trabajo de género. De esta forma será más factible el análisis y facilitara la respuesta a la interrogante planteada al inicio del trabajo.

\section{Análisis del número total de niños/as y adolescentes ecuatorianas que realizan trabajo infantil en relación con el número de mujeres ecuatorianas que han sufrido violencia de género.}


Tabla 3. Total de niños/as y adolescentes que trabajan en relación con mujeres que sufren de violencia de género

\begin{tabular}{|c|c|c|}
\hline Provincia & Total de niños que trabajan (X) & Mujeres Maltratadas (Y) \\
\hline Amazonía & 259.381 & 228.443 \\
\hline Azuay & 206.483 & 258.057 \\
\hline Bolívar & 58.424 & 56.541 \\
\hline Cañar & 71.084 & 91.641 \\
\hline Carchi & 46.713 & 49.271 \\
\hline Chimborazo & 136.894 & 137.289 \\
\hline Cotopaxi & 127.503 & 127.822 \\
\hline El Oro & 170.898 & 174.223 \\
\hline Esmeraldas & 179.711 & 154.252 \\
\hline Guayas & 1.011 .284 & 1.035 .536 \\
\hline Imbabura & 117.895 & 129.908 \\
\hline Loja & 134.142 & 134.165 \\
\hline Los Ríos & 238.979 & 217.749 \\
\hline Manabí & 408.711 & 328.672 \\
\hline Pichincha & 674.481 & 940.250 \\
\hline Santa Elena & 94.537 & 71.512 \\
\hline Santo Domingo & 115.987 & 109.123 \\
\hline Tungurahua & 134.985 & 183.938 \\
\hline
\end{tabular}

Tabla elaborada por la autora; datos obtenidos de (Instituto Ecuatoriano de Estadísticas y Censos, 2012).

Una vez realizado el modelo de regresión lineal para las variables dependientes e independientes, se obtiene la siguiente tabla con los siguientes datos y la gráfica:

Tabla 4. Resumen del modelo de regresión lineal del primer análisis

\begin{tabular}{lr}
\hline \multicolumn{2}{c}{ Estadísticas de la regresión } \\
\hline Coeficiente de correlación múltiple & 0,97 \\
Coeficiente de determinación $\mathrm{R}^{\wedge} 2$ & 0,95 \\
$\mathrm{R}^{\wedge} 2$ ajustado & 0,94439309 \\
Error típico & 65963,4772 \\
Observaciones & 18 \\
\hline
\end{tabular}

Tabla elaborada por la autora 
Gráfico 1. Curva de regresión ajustada de las variables "total de niños/as y adolescentes que son obligados a trabajar y total de mujeres que sufren maltrato de género"

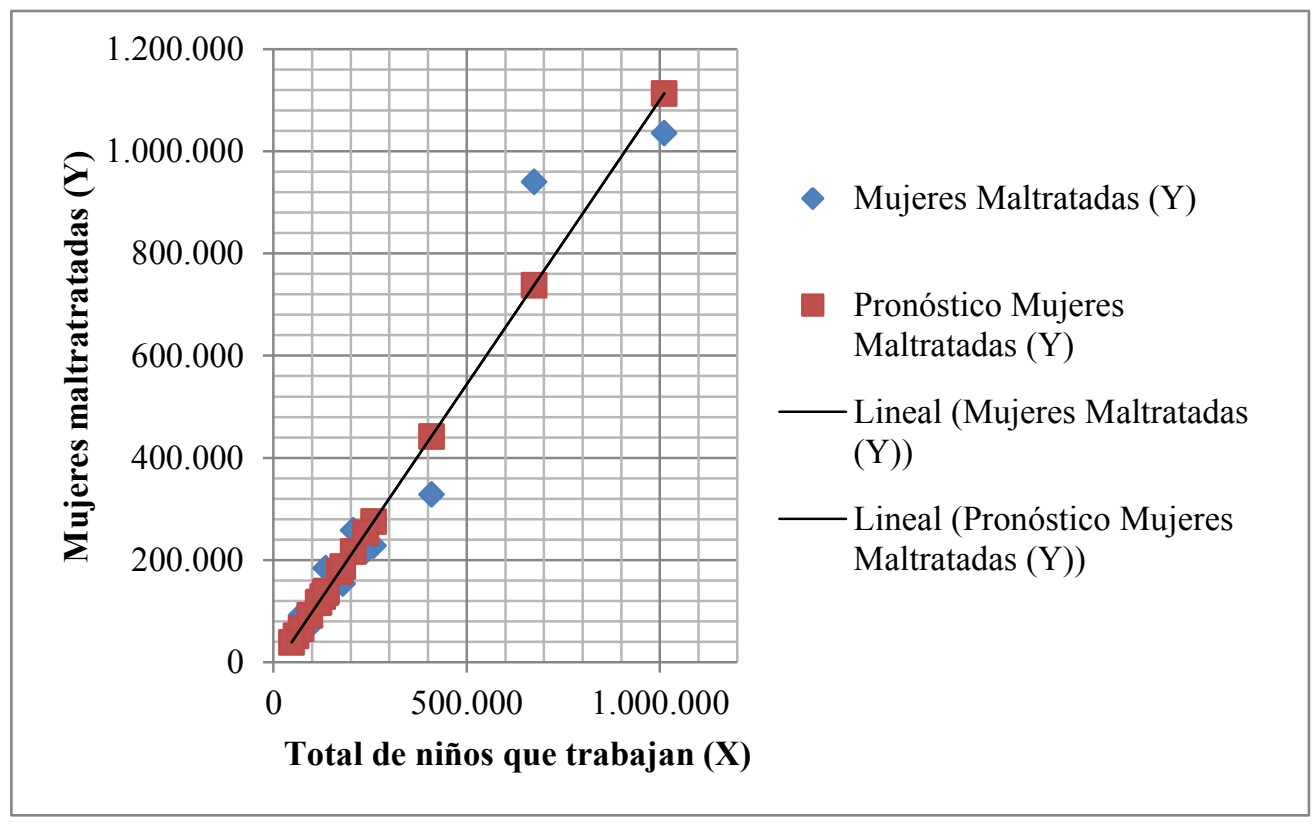

Gráfico elaborado por la autora

En el primer análisis se consideran todas las variables que aparecen mencionadas en la tabla 6. El coeficiente de determinación $\mathrm{R}^{\wedge} 2=0.9477$, lo cual indica que cae en una correlación fuerte con $95 \%$ y que la relación del total de niños/as y adolescentes ecuatorianos de entre 5 a 17 años que se encuentran trabajando en comparación con el número de mujeres que sufre violencia de género es fuerte al superar el 0.75 de una correlación fuerte, por ende el trabajo infantil si tiene una fuerte influencia en el maltrato a la mujer a un futuro, sin embargo es muy importante denotar que este problema social no solo viene de la mano con el maltrato infantil, ya que hay una gran cantidad de variables que también intervienen, tales como: los patrones de conducta aprendidos en los hogares por los niños a través de sus padres y que se traspasan, en la mayoría de los casos, de generación en generación; las normas sociales que tratan de minimizar el daño ocasionado por el cónyuge y muchas veces justificarlo "estas normas intentan explicar la actuación atribuyéndola a trastornos del conyugue o, incluso, de la 
mujer, excusando al hombre de padecer problemas de estrés, de alcohol, de personalidad" (Burriel, s.f.), entre otras más.

Por otro lado en el gráfico de dispersión si se cumple la relación lineal entre las variables Trabajo infantil y violencia de género.

Tabla 5. Intersección de las variables: niños que trabajan y mujeres maltratadas

\begin{tabular}{|l|l|r|r|}
\cline { 2 - 4 } \multicolumn{1}{c|}{} & & \multicolumn{1}{c|}{ Coeficientes } & \multicolumn{1}{c|}{ Error típico } \\
\hline $\mathbf{B}_{\mathbf{1}}$ & Intercepción & $-13036,55$ & 21757,205 \\
\hline $\mathbf{B}_{\mathbf{0}}$ & Total de niños que trabajan $(\mathrm{X})$ & 1,11 & 0,0654134 \\
\hline
\end{tabular}

Tabla elaborada por la autora

$$
y^{\wedge}=\beta_{0}+\beta_{1} x
$$

A partir de los coeficientes de la regresión obtenidos, se podría establecer un modelo determinando coeficientes estimados para $\beta_{0}$ (conocida como constante de la recta) y para $\beta 1$ (pendiente de la recta); sin embargo esto sería factible si se buscara generar una ecuación de regresión para comparar la dependencia de una variable con la otra en diferentes proporciones o valores dados a la variable dependiente (X), pero dado el objetivo del estudio, este paso es irrelevante en la investigación la cual solo busca responder una interrogante.

Análisis del número de niños ecuatorianos que realizan trabajo infantil en relación con el número de mujeres ecuatorianas que han sufrido violencia de género. 
Tabla 6. Total de niños y adolescentes ecuatorianos de género masculino que trabajan en relación con mujeres que sufren de violencia de género

\begin{tabular}{|c|c|c|}
\hline Provincia & Niños que trabajan (X) & Mujeres Maltratadas (Y) \\
\hline Amazonía & 133.117 & 228.443 \\
\hline Azuay & 104.366 & 258.057 \\
\hline Bolívar & 30.289 & 56.541 \\
\hline Cañar & 34.971 & 91.641 \\
\hline Carchi & 23.405 & 49.271 \\
\hline Chimborazo & 71.165 & 137.289 \\
\hline Cotopaxi & 63.850 & 127.822 \\
\hline El Oro & 88.770 & 174.223 \\
\hline Esmeraldas & 92.266 & 154.252 \\
\hline Guayas & 123.985 & 1.035 .536 \\
\hline Imbabura & 58.658 & 129.908 \\
\hline Loja & 70.257 & 134.165 \\
\hline Los Ríos & 123.985 & 217.749 \\
\hline Manabí & 208.667 & 328.672 \\
\hline Pichincha & 337.372 & 940.250 \\
\hline Santa Elena & 48.829 & 71.512 \\
\hline Santo Domingo & 59.407 & 109.123 \\
\hline Tungurahua & 69.588 & 183.938 \\
\hline
\end{tabular}

Tabla hecha por la autora-datos obtenidos por (Instituto Ecuatoriano de Estadísticas y Censos, 2012)

Una vez empleado el modelo de regresión lineal para las variables, se obtiene el siguiente gráfico de dispersión y los siguientes datos:

Tabla7. Resumen del modelo de regresión lineal del segundo análisis

\begin{tabular}{lr}
\hline \multicolumn{2}{c}{ Estadisticas de la regresión } \\
\hline Coeficiente de correlación múltiple & 0,74 \\
Coeficiente de determinación $\mathrm{R}^{\wedge} 2$ & 0,55 \\
$\mathrm{R}^{\wedge} 2$ ajustado & 0,5182818 \\
Error típico & 194149,223 \\
Observaciones & 18 \\
\hline
\end{tabular}

Tabla elaborada por la autora 
Gráfico 2. Curva de regresión ajustada de las variables "total de niños y adolescentes varones que son obligados a trabajar y total de mujeres que sufren maltrato de género"

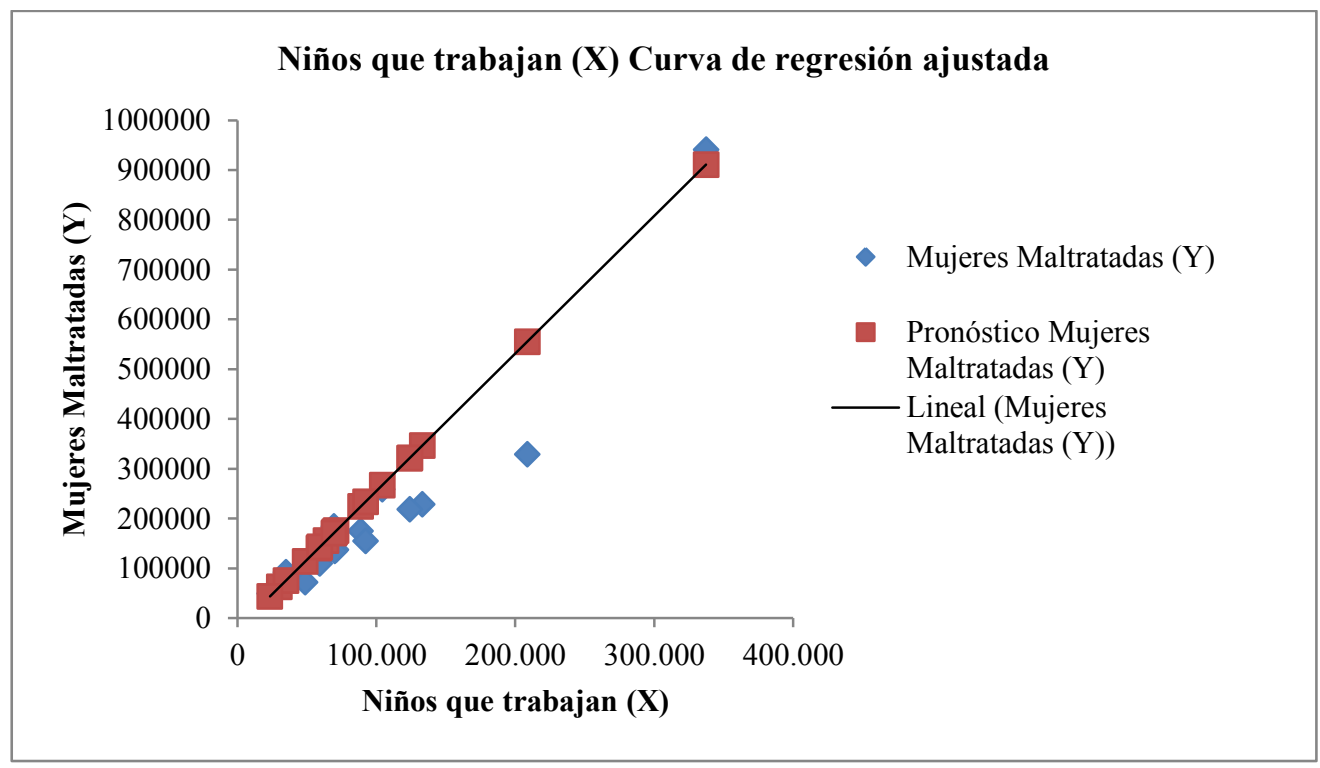

Gráfico elaborado por la autora

Tabla 8. Intersección de las variables: niños que trabajan y mujeres maltratadas

\begin{tabular}{|c|c|ccc}
\cline { 2 - 3 } \multicolumn{2}{c}{} & \multicolumn{1}{c}{ Coeficientes } & Error típico & Estadístico t \\
\hline B0 & Intercepción & $-21439,6813$ & 76173,9827 & $-0,281456746$ \\
B1 & Niños que trabajan (X) & 2,76217005 & 0,62889834 & 4,392077203 \\
\hline
\end{tabular}

Tabla elaborada por la autora

En el segundo análisis se consideran todas las variables que aparecen mencionadas en la tabla 5. El coeficiente de determinación $\mathrm{R}^{\wedge} 2=0.5466$, lo cual indica que cae en una correlación débil con $54.66 \%$ y que la relación del total de niños y adolescentes ecuatorianos de entre 5 a 17 años y de sexo masculino que se encuentran trabajando en comparación con el número de mujeres que sufre violencia de género es débil al no superar el 0.75 de una correlación fuerte, por ende el trabajo infantil no influye en gran medida en el maltrato a la mujer a un futuro por 
parte del género masculino; sin embargo hay mas factores que ponen a los hombres en primera instancia como los promotores de la agresión femenina, ya que son ellos los que dieron inicio a este mal social oponiéndose a la participación de la mujer en la sociedad democrática y poniendo trabas para evitar una convivencia en igualdad de derechos.

Análisis del número de niñas y adolescentes ecuatorianas que realizan trabajo infantil en relación con el número de mujeres ecuatorianas que han sufrido violencia de género

Tabla 9. Niñas y adolescentes ecuatorianas de género femenino en relación con mujeres que sufren de violencia de género

\begin{tabular}{|c|c|c|}
\hline Provincia & Niñas que trabajan $(\mathbf{X})$ & Mujeres Maltratadas (Y) \\
\hline Amazonía & 126.264 & 228.443 \\
\hline Azuay & 102.117 & 258.057 \\
\hline Bolívar & 28.135 & 56.541 \\
\hline Cañar & 36.114 & 91.641 \\
\hline Carchi & 23.308 & 49.271 \\
\hline Chimborazo & 65.730 & 137.289 \\
\hline Cotopaxi & 63.653 & 127.822 \\
\hline El Oro & 82.128 & 174.223 \\
\hline Esmeraldas & 87.445 & 154.252 \\
\hline Guayas & 496.231 & 1.035 .536 \\
\hline Imbabura & 59.237 & 129.908 \\
\hline Loja & 63.885 & 134.165 \\
\hline Los Ríos & 114.994 & 217.749 \\
\hline Manabí & 200.044 & 328.672 \\
\hline Pichincha & 337.110 & 940.250 \\
\hline Santa Elena & 45.708 & 71.512 \\
\hline Santo Domingo & 56.581 & 109.123 \\
\hline Tungurahua & 65.398 & 183.938 \\
\hline
\end{tabular}

Tabla hecha por la autora-datos obtenidos por (Instituto Ecuatoriano de Estadísticas y Censos, 2012) 
Una vez empleado el modelo de regresión lineal para las variables, se obtiene el siguiente gráfico y los siguientes datos:

Tabla 10. Resumen del modelo de regresión lineal del tercer análisis

\begin{tabular}{lr}
\hline \multicolumn{2}{c}{ Estadisticas de la regresión } \\
\hline Coeficiente de correlación múltiple & 0,98 \\
Coeficiente de determinación $\mathrm{R}^{\wedge} 2$ & 0,95 \\
$\mathrm{R}^{\wedge} 2$ ajustado & 0,94967724 \\
Error típico & 62751,1068 \\
Observaciones & 18 \\
\hline
\end{tabular}

Tabla elaborada por la autora

Gráfico 3. Curva de regresión ajustada de las variables "total de niñas y adolescentes de género femenino que son obligadas a trabajar y total de mujeres que sufren maltrato de género"

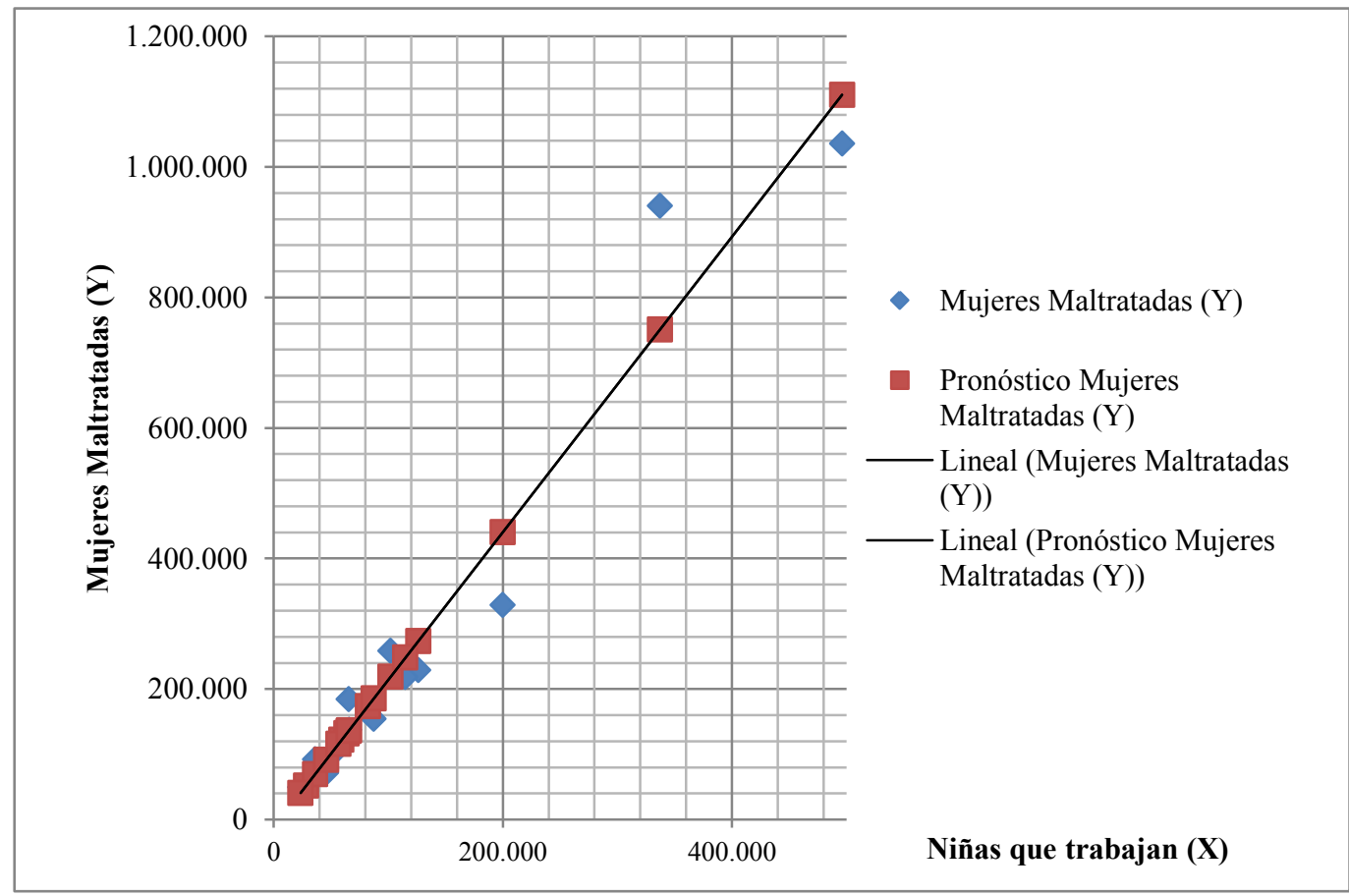

Gráfica elaborada por la autora

Cuestiones de género: de la igualdad y la diferencia - e-I.S.S.N: 2444-0221 - No 10, 2015 - pp. 376-393 
Tabla 11. Intersección de las variables: niñas que trabajan y mujeres maltratadas

\begin{tabular}{|l|l|rrr}
\cline { 5 - 5 } \multicolumn{2}{c}{} & \multicolumn{1}{c}{ Coeficientes } & Error tipico & \multicolumn{1}{c}{ Estadístico $t$} \\
\hline $\mathbf{B}_{\mathbf{0}}$ & Intercepción & $-12112,92$ & 20635,256 & $-0,587001168$ \\
\hline $\mathbf{B}_{\mathbf{1}}$ & Niñas que trabajan $(\mathrm{X})$ & 2,26 & 0,12609428 & 17,93932252 \\
\hline
\end{tabular}

Tabla elaborada por la autora

En el tercer análisis se consideran todas las variables que aparecen mencionadas en la tabla 9. El coeficiente de determinación $\mathrm{R}^{\wedge} 2=0.9526$, lo cual indica que cae en una correlación fuerte con $95 \%$ y que la relación del total de niñas y adolescentes ecuatorianas de entre 5 a 17 años que se encuentran trabajando en comparación con el número de mujeres que sufren violencia de género por provincia es muy fuerte al superar el 0.75 de una correlación fuerte, por ende el trabajo infantil en las niñas si influye en gran medida en el maltrato a la mujer a un futuro, pero como se mencionó anteriormente existen más variables que influyen en este problema.

\section{Conclusiones}

Los resultados obtenidos a través de estos tres análisis indican que el trabajo infantil si puede ser considerado como una variable que influye en el maltrato de género en gran medida, sin embargo tiene más influencia en el género femenino puesto que las niñas y adolescentes que si han sido obligadas a trabajar, en un futuro tenderán a sentirse objeto de abuso por parte de su conyugue y sufrirán maltratos en el transcurso de su desarrollo al haberse criado en un ambiente de abuso continuo por parte de sus familiares o terceros.

Comparando los resultados con los análisis anteriores las niñas tienden a sufrir más los efectos del abuso al ser más vulnerables en comparación a los niños, independientemente de que el porcentaje de trabajo infantil sea mayor en los varones ya que un menor porcentaje de niñas que son obligadas a laborar entre los 5 a 17 años de edad, ellas tienden a sufrir más maltrato al crecer con una mentalidad de abuso e inferioridad. Por otro lado los niños crecen 
con mentalidad machista y desahogan su enojo y años de maltrato de sus futuras esposas, generando de esta manera un gran índice de violencia de género en el país.

Las variables relacionadas al maltrato y que sirven como base para que este se genere y siga creciendo, sin innumerables, sin embargo se podría denominar "la relación de desigualdad entre hombres y mujeres" como la más destacada y que data desde siglos atrás, épocas donde las mujeres eran consideradas entes carente de valor alguno, eran objeto de posesión, y eran consideradas como una mercancía que se podía vender, poseer o alquilar para los hombres.

La violencia contra la mujeres es considerada estructural "La violencia contra las mujeres se produce en una sociedad que mantiene un sistema de relaciones de género que perpetúa la superioridad de los hombres sobre las mujeres y asigna diferentes atributos, roles y espacios en función del sexo" (Burriel, s.f.); y ambas partes (tantos hombres como mujeres) han contribuido para que aun crezca y se mantenga aun impotente en esta sociedad en pleno siglo XXI.

Son muchos los factores que engloban esta relación, siendo uno de estos la cultura en sí, ya que en el Ecuador aún se pueden apreciar rasgos de machismo en muchos de sus ciudadanos. "La cultura ha legitimado la creencia de la posición superior del varón, lo cual ha facilitado que las mujeres se sientan inferiores” (Expósito, 2005).

Es importante destacar que cuando una niña a temprana edad es puesta como objeto de abusos y se cría en un ambiente de continuo maltrato, explotación, miedo, inseguridad y violencia psicológica, en el futuro crecerá como una persona carente de autoestima y llena de miedo e inseguridad hacia la sociedad, lo cual facilitara el hecho de que se convierta nuevamente en un objeto de abusos y maltrato por parte de su conyugue. Las acciones del pasado repercuten en el desarrollo integral de un individuo a futuro; y si el trabajo infantil se sigue dando en el país es muy probable que la tasa de mujeres que son víctimas del maltrato crezca y así como también la tasa de femicidios. 


\section{BIBLIOGRAFÍA}

- Burriel, Jose Antonio. (s.f.): “Cause de la violencia de género", [en línea] Disponible en: http://nomasvg.com/informacion-sobre-violencia-de-genero/causa-de-la-violencia-de-genero/ [05/08/2014].

- Consejo Nacional de Población (2012): " Violencia emocional, física, sexual y económica", [en línea] Disponible en: http://www.violenciaenlafamilia.conapo.gob.mx/en/Violencia_Familiar/Violencia_emocional fsica_sexual_y econmica [05/08/2014].

- Expósito, Francisca (2011): "Violencia de Género". En: Investigación y Ciencia, № 48, [en línea] Disponible en: $\underline{\text { http://www.investigacionyciencia.es/revistas/mente-y- }}$ cerebro/numero/48/violencia-de-gnero-8894 [06/08/2014].

- Humanium - ayuda a los niños (2014): "Trabajo infantil", [en línea] Disponible en: http://www.humanium.org/es/trabajo-infantil/ [21/08/2014].

- Instituto Ecuatoriano de Estadisticas y Censos (2011): "Encuesta Nacional de Relaciones Familiares y Violencia de Género contra las Mujeres", [en línea] Disponible en: http://anda.inec.gob.ec/anda/index.php/catalog/94 [18/08/2014].

- Instituto Ecuatoriano de Estadísticas y Censos (2012): " Encuesta Nacional de Relaciones Familiares y Violencia de Género contra las Mujeres ", [en línea] Disponible en: http://www.humanas.org.ec/pdf/Violencia_de_Genero_Ecuador_encuesta_nacional.pdf [11/08/2014].

- Organización de las Naciones Unidas (1993): "Declaración sobre la eliminación de la violencia contra la mujer", [en línea] Disponible en: http://www.ohchr.org/SP/ProfessionalInterest/Pages/ViolenceAgainstWomen.aspx [12/08/2014].

- UNICEF (2014): "Trabajo infantil", [en línea] Disponible en: http://www.unicef.org/spanish/protection/index_childlabour.html [08/07/2014].

- Vasconez, Alison (2003): "Niñez a Medias, Futuro a Medias- Trabajo Infantil y Juvenil en el Ecuador", [en línea] Disponible en: http://www.flacso.org.ec/docs/av ninesamedias.pdf [17/07/2014]. 